\title{
Pathologie thyroïdienne morphologique radio-induite par de faibles doses d'irradiation cervicale : apport d'une enquête épidémiologique de cohorte
}

\author{
Philippe Fragu, Florent De Vathaire, Pascal François, Marie-Françoise Avril
}

\section{Summary}

Morphological thyroid abnormalities after cervical irradiation with low doses : interest of an epidemiological study on a single cohort population

Thyroid morphological exploration was carried out on 396 patients who were recalled because their thyroid gland had been exposed during angiomas irradiation in childhood 11.43 years before (median, 22 years). Among these were 24 patients with a simple goiter and 13 patients with a single thyroid nodule. The irradiations have been classified in two categories based on their duration : short duration, from a few seconds to a few minutes $\left({ }^{90} \mathrm{Sr}\right.$, Xrays) and long duration $\left({ }^{226} \mathrm{Ra},{ }^{32} \mathrm{P},{ }^{90} \mathrm{Y}\right)$, from 30 minutes to several hours. The risk of a thyroid nodule increased significantly with the total dose received by the thyroid ; it was linked to the dose delivered in the short duration but not to that delivered in the long duration. The risk of a goiter, which also increased with the dose received by the thyroid, did not depend on the duration of the irradiation. This study emphasizes the role of the dose rate in the risk of thyroid nodule.
L'utilisation de faibles doses de radiations ionisantes $(<1$ Gy) à proximité de la thyroïde risque-t-elle, à terme, d'entraîner des désordres thyroïdiens? L'étude d'une population cohorte de 396 patients, irradiés pour un angiome cutané dans l'enfance (recul moyen 22 ans), apporte des informations importantes sur le sujet. Cette étude a été rendue possible grâce à l'existence d'un dossier unique qui regroupait les données médicales et techniques pour chaque patient.

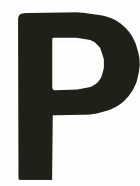

lusieurs études épidémiologiques effectuées après irradiation externe de la glande thyroïde [1] ont établi que le risque de nodule ou de cancer thyroïdien est, pour une même dose, plus important lorsque l'irradiation survient dans l'enfance ou chez une femme ; ce risque peut se manifester très longtemps après l'irradiation. Pour les irradiations à fort débit, entre $100 \mathrm{mGy}$ et au moins $10 \mathrm{~Gy}$, la relation entre le risque et la dose délivrée à la thyroïde est linéaire. Par ailleurs, aucune augmentation significative du risque de tumeurs thyroïdiennes n'a été obser- vée après administration d'activité diagnostique ou thérapeutique d'iode $131[2,3]$. En revanche, le devenir des sujets irradiés des îles Marshall après l'explosion atomique de Bikini suggère que les isotopes de l'iode à demi-vic brève ont un effet tumorigène plus important que l'iode 131 , ce qui est attribué à un débit de dose plus élevé [4].

Cependant, l'effet du débit de dose n'a jamais été analysé dans une même cohorte de sujets irradiés à des débits de dose suffisamment variables. En effet, cette étude implique des conditions difficiles à réunir, comme le recrutement d'une population exposée suffisamment nombreuse et irradiée au moins dix ans auparavant, des informations fiables sur les traitements et les doses administrées. A l'Institut Gustave-Roussy (Villejuif, France), plus de 5000 enfants porteurs d'un angiome cutané ont été traités entre 1941 et 1973 par différentes techniques de radiothérapie ayant des débits de dose très variables [5]. Cela nous a amenés à proposer un programme de reconvocation de ces malades afin d'évaluer les séquelles à long terme de ces traitements [6]. Parmi les 1137 patients irradiés qui ont répondu à ce programme, 396 enfants (305 filles et 91 garçons) ont eu leur thyroïde exposée en raison soit de la proximité de l'angiome (moins de $5 \mathrm{~cm}$ pour une irradiation $\beta$ pure), soit du type d'irradiation $(\gamma$ ou X) ; $95 \%$ d'entre eux ont été irradiés au cours de leur première année. Cette cohorte nous a permis d'étudier l'effet du débit de dose sur le risque de tumeur thyroïdienne radio-induite. 


\section{Dosimétrie}

Les angiomes cutanés ont été, pendant de nombreuses années, traités par curiethérapie avec ${ }^{226} \mathrm{Ra}$ (plaques ou aiguilles). Ce traitement a été progressivement remplacé par une curiethérapie utilisant un radioélément émetteur $\beta$ pur (applicateur de ${ }^{90} \mathrm{Sr}$ ou de ${ }^{32} \mathrm{P}$, aiguille de $\left.{ }^{90} \mathrm{Y}\right)$. Certains de ces angiomes ont été traités par radiothérapie externe (contacthérapie et téléradiothérapie). Les indications thérapeutiques dépendaient de la taille et de l'épaisseur de l'angiome [7].

Disposant pour chaque patient du dossier technique de l'irradiation, il a été possible de calculer la dose délivrée à la thyroïde. La distance entre l'angiome cutané et la thyroïde a tout d'abord été estimée à l'aide d'un modèle mathématique, en tenant compte de la taille de chaque enfant [8]. Pour chaque radioélément, la dose reçue par la thyroïde a été ensuite évaluée en utilisant des tables [9] qui estiment la dose en fonction de l'activité et de la distance source (localisation de l'angiome)cible (thyroïde). Pour les traitements par radiothérapie externe, la dose délivrée à la thyroïde a été évaluée à l'aide d'un modèle d'estimation de dose délivrée dans et à distance du champ d'irradiation [10]. La figure 1 montre les doses reçues par la thyroïde pour chaque type d'irradiation. Il est à noter que ces doses sont faibles puisque $98 \%$ d'entre elles sont inférieures à $1 \mathrm{~Gy}$.

Certains enfants ont été irradiés avec plusieurs appareils qui ont pu être utilisés pendant plusieurs séances avec des durées variables. Cela nous a conduits à regrouper les traitements en deux catégories: traitements de courte durée et fort débit, de quelques secondes à quelques minutes $\left({ }^{90} \mathrm{Sr}\right.$, rayons $\left.\mathrm{X}\right)$ d'une part, et traitements de longue durée et débit plus faible, de 30 minutes à quelques heures $\left({ }^{226} \mathrm{Ra},{ }^{32} \mathrm{P},{ }^{90} \mathrm{Y}\right)$ d'autre part. Le rapport des débits de dose entre les deux groupes est d'environ un facteur 30 (Tableau I).

\section{Modèle statistique}

La relation débit de dose-désordres 972 diée à l'aide d'un modèle de régression poissonienne dans lequel l'excès de risque relatif de nodule ou de goitre par personne et par an était une fonction linéaire de la dose. Le modèle tenait compte du sexe et était stratifié sur la durée de surveillance entre l'irradiation et l'examen. Les différents modèles utilisés étaient les suivants :

(0) risk $=H_{0}$

(1) risk $=H_{0} \exp (\beta$ sexe $)$

(2) risk $=H_{0}(1+\alpha$ dose $) \exp (\beta$ sexe $)$

(3) risk $=H_{0}(1+\alpha 1$ dose $1+\alpha 2$ dose 2) $\exp (\beta$ sexe $)$

Dans ces modèles $H_{0}$ est l'incidence spontanée de nodules ou goitres ; $\alpha$, $\alpha 1, \alpha 2$ et $\beta$ sont les coefficients du modèle; dose, dose 1 et dose 2 sont respectivement la dose totale, la dose délivrée avec un fort débit et la dose délivrée avec un faible débit. La différence de déviance entre les modèles 1 et 2 mesure la significativité statistique de l'effet dose, et celle entre les modèles 2 et 3 la significativité de l'effet débit de dose.

\section{Relation débit de dose-nodule thyroïdien}

Les 396 patients de cette cohorte ont été surveillés en moyenne 22 ans ; ils ont apporté 8603 personnes-années et 793 personnes-années-grays d'observation. Au terme d'un bilan clinique et scintigraphique, 38 patients étaient porteurs d'une anomalie morphologique thyroïdienne (Tableau I). Chez 24 d'entre eux, il s'agissait d'un goitre simple, soit un taux d'incidence absolu de 30 pour 1000 personnes par an et par Gy. Quatorze enfants ont développé un nodule thyroïdien unique (treize bénins et un cancer papillaire) ; cinq de ces nodules avaient été découverts avant le bilan clinique effectué à l'IGR, et neuf lors de ce bilan. Le risque cumulé de nodules 30 ans après l'irradiation était de $6 \%$ (intervalle de confiance à $95 \%$ : $1,6 \%-8,9 \%)$ et le taux d'incidence absolu de 1,8 pour 1000 personnes par an et par Gy. Aucune influence du sexe n'a été observée sur le risque de goitre; en revanche, le risque de nodule était trois fois plus élevé parmi les femmes $\left(\chi^{2}=2,8, \mathrm{ddl}=\right.$ $1, \mathrm{p}=0,1)$.

Pour ces deux anomalies morphologiques, l'excès de risque augmentait avec la dose : le risque relatif par gray était de 3 pour les nodules $\left(\chi^{2}\right.$

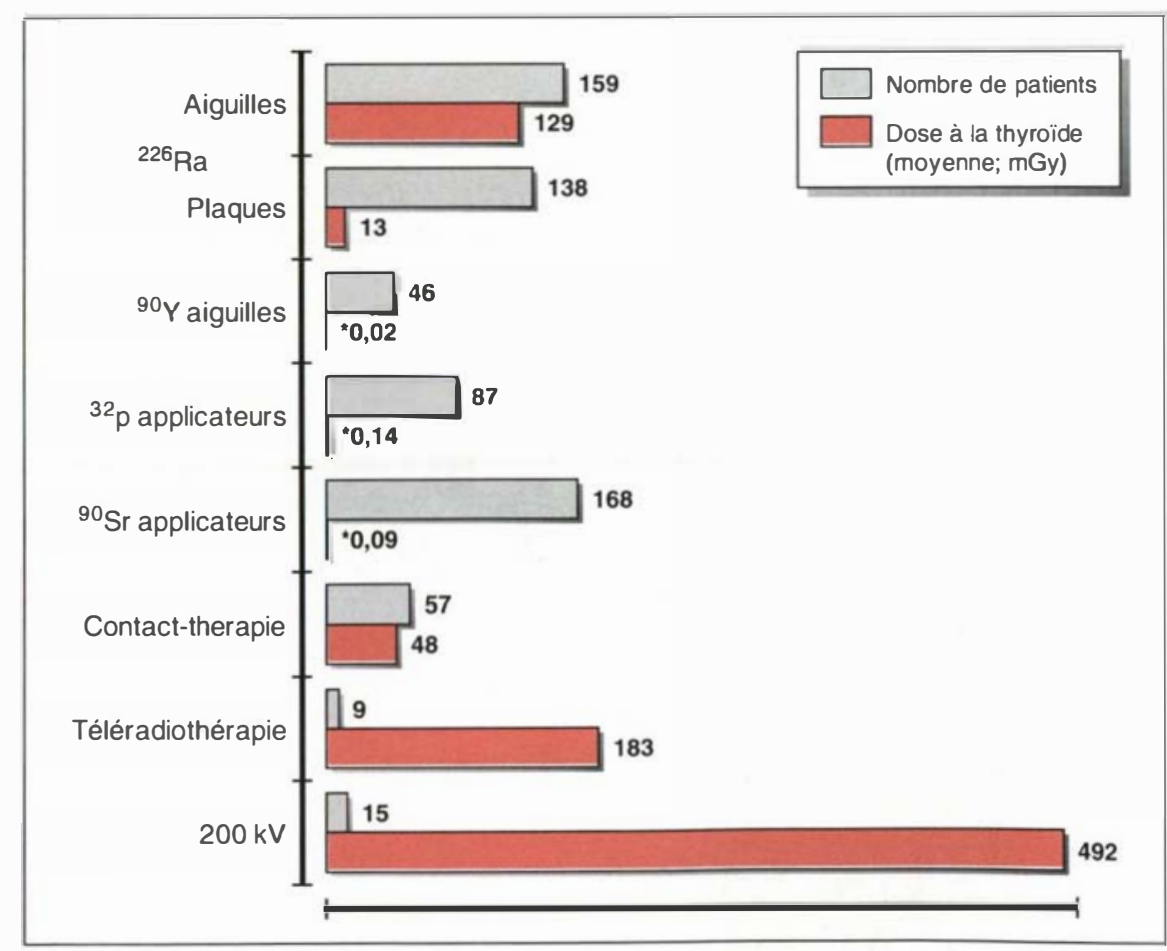

Figure 1. Dose délivrée à la thyroïde en fonction des modalités d'irradiation de l'angiome cutané (1941-1973). 


\section{1. Épidémiologie [14]}

Cohorte : ensemble des individus répondant à des caractéristiques initiales précises. Une cohorte peut être prospective ou rétrospective.

Nombre de personnes-années (ou person-year) d'observation (PY) : ce nombre est la somme des suivis, exprimés en années, des sujets de la cohorte pendant une période donnée.

Nombre de personnes-années-gray (ou person-year-gray) d'observation (PYG): c'est la somme des durées de suivi de chaque sujet irradié, multipliée par la dose reçue par chaque sujet exprimée en grays.

Estimation de l'incidence de nodule ou de goitre : on définit le taux d'incidence par période comme le rapport entre le nombre de nodules ou de goitres diagnostiqués et le nombre de PY. L'incidence cumulée sur plusieurs périodes se calcule à partir des incidences sur chaque période, à l'aide des méthodes utilisées pour les données de survie; méthode actuarielle ou de Kaplan-Meier.
Indices de risque : l'indice de risque le plus communément utilisé est le risque relatif $(R R)$. C'est le rapport entre le nombre observé de nodules ou de goitres dans le groupe de sujets irradiés et le nombre attendu dans un groupe non irradié. Un autre indice très utilisé est l'excès de risque (absolute excess risk : $A E R$ ). C'est la différence entre l'incidence de nodules ou de goitres observés dans le groupe irradié et celle attendue dans le groupe non irradié. Ces deux indices peuvent être rapportés à la dose de radiations reçue.

\section{Radiothérapie}

Gray (Gy) : c'est l'unité (SI) de dose absorbée de rayonnement ionisant exprimée en joule par unité de masse exprimée en kilogramme. Un Gray est égal à 100 rads.

Radium : ${ }^{226} R a$ émetteur $\alpha$ de 4,78 $\mathrm{MeV}$ et de $\gamma$ d'énergie multiple, période de désintégrations 1622 ans; Strontium : ${ }^{90} \mathrm{Sr}$ émetteur $\beta^{-}$-d'énergie maximale de $0,546 \mathrm{MeV}$, période 28,1 ans; Phosphore: ${ }^{32} \mathrm{P}$ émetteur $\beta$ - de $1,710 \mathrm{MeV}$, période 14,3 jours ; Yttrium : ${ }^{90} Y$ émetteur $\beta^{-}$de 2,283 $\mathrm{MeV}$, période 2,7 jours.

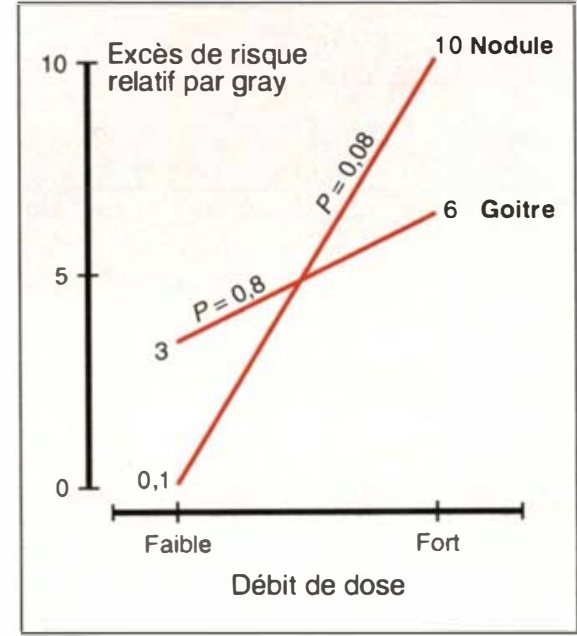

Figure 2. Effet du débit de dose sur la pathologie morphologique thyroidienne: goitre simple et nodule thyroïdien.

Tableau I

ANOMALIE MORPHOLOGIQUE OBSERVÉE DANS UNE COHORTE DE 396 PATIENTS IRRADIÉS DANS L'ENFANCE POUR UN ANGIOME CUTANÉ

\begin{tabular}{|c|c|c|c|}
\hline & \multicolumn{3}{|c|}{ Type d'irradiation } \\
\hline & $\begin{array}{l}\text { Longue durée } \\
\text { Faible débit* } \\
N=118\end{array}$ & $\begin{array}{l}\text { Courte durée } \\
\text { Fort débit** } \\
N=103\end{array}$ & $\begin{array}{l}\text { Association } \\
\text { des } 2 \text { types } \\
N=115\end{array}$ \\
\hline $\begin{array}{l}\text { Sexe } \\
\text { Garçon/Fille }\end{array}$ & $45 / 133$ & $24 / 79$ & $22 / 93$ \\
\hline $\begin{array}{l}\text { Année de traitement } \\
\text { Médiane } \\
\text { Extrêmes }\end{array}$ & $\begin{array}{c}1962 \\
1943-1972\end{array}$ & $\begin{array}{c}1968 \\
1955-1973\end{array}$ & $\begin{array}{c}1964 \\
1946-1973\end{array}$ \\
\hline $\begin{array}{l}\text { Dose thyroïde Gy } \\
\text { Moyenne } \\
\text { Extrême }\end{array}$ & $\begin{array}{c}0,094 \\
<0,00001\end{array}$ & $\begin{array}{c}0,075 \\
<0,00001-2,74\end{array}$ & $\begin{array}{c}0,084 \\
<0,00001-0,87\end{array}$ \\
\hline $\begin{array}{l}\text { Débit de dose thyroïde } \\
\text { moyenne }(\mathrm{Gy} / \mathrm{s})\end{array}$ & $5.10^{-6}$ & $138.10^{-6}$ & $5.10^{-6}$ \\
\hline Goitre observé & 8 & 8 & 8 \\
\hline Nodule observé & 1 & 4 & 9 \\
\hline
\end{tabular}

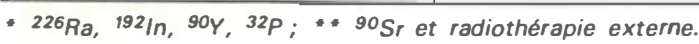


$=4,3, \mathrm{ddl}=1, \mathrm{p}=0,04)$ et de 4 pour les goitres $\left(\chi^{2}=5,2, \mathrm{ddl}=, \mathrm{p}\right.$ $=0,02)$. La figure 2 montre l'effet du débit de dose. Il n'affecte pas le risque de goitre. En revanche, le risque de nodule était augmenté dans le groupe de sujets irradiés avec un fort débit $\left(\chi^{2}=3,1, \mathrm{ddl}=1, \mathrm{p}=\right.$ $0,08)$ tandis qu'il n'était pas modifié dans le groupe irradié avec un faible débit.

\section{Discussion}

Malgré la dimension modérée de notre cohorte, nous apportons une contribution originale à l'étude du débit de dose sur l'incidence des tumeurs thyroïdiennes radio-induites. En effet, ce problème n'avait été abordé jusqu'à maintenant dans la littérature qu'en comparant les résultats d'études épidémiologiques réalisées sur des populations différentes et irradiées dans des conditions techniques souvent totalement opposées. Notre étude épidémiologique rétrospective, qui porte sur une population homogène de 396 patients, irradiés pour un angiome cutané dans l'enfance, apporte des informations importantes sur les séquelles thyroïdiennes à long terme de faibles doses de radiations ionisantes $(<1 \mathrm{~Gy})$. Elle souligne le rôle du débit de dose dans l'apparition de la pathologie nodulaire thyroïdienne.

Malgré le petit nombre de nodules observés, il existe une augmentation significative du risque de leur apparition, uniquement pour des irradiations de courte durée et à fort débit. Ce résultat est en accord avec ceux de Ron et al. [11], qui ont observé que le risque de cancer thyroïdien est augmenté d'un facteur 4 pour une dose moyenne de 0,09 Gy délivrée par radiothérapie externe. De même, l'absence d'augmentation de risque de nodule thyroïdien, pour les irradiations de longue durée à faible débit, confirme les observations effectuées après utilisation diagnostique de l'iode 131 (même pour des activités entraînant des doses de $0,5 \mathrm{~Gy}$ à la thyroïde) ou dans les zones à fort taux d'irradiation naturelle $(1,8$ à 2,8 mGy/an à la thyroïde). Dans ces deux cas, il n'a pas été mis en évidence d'augmentation significative du
Un autre résultat de notre étude peut paraître a priori surprenant : il concerne la relation dose thyroïde-goitre. Il est à rapprocher des observations effectuées dans des régions à haut niveau de radio-activité naturelle [12]. Par ailleurs, il ne faut pas oublier que la France est un pays où l'apport quotidien iodé est faible (environ $100 \mu \mathrm{g}$ par jour), ce qui favorise l'apparition d'une hypertrophie thyroïdienne pour assurer une hormonosynthèse normale. Il est intéressant de noter que le contenu en iode de ces thyroïdes irradiées est significativement abaissé et que cette diminution est significativement liée à la dose totale reçue par la thyroïde [13]. L'extrême radiosensibilité du tissu thyroïdien de ces enfants, dont la grande majorité a été irradiée dans la première année de leur vie, pourrait expliquer l'apparition de ces goitres. Cette étude a été rendue possible en raison de l'existence d'un dossier unique qui regroupait les données médicales et techniques (modalités d'irradiation, photographie initiale de l'angiome) pour chaque patient. Grâce à une gestion centralisée dans un service d'archives médicales, les informations sont ainsi toujours accessibles, y compris pour des patients examinés 40 ans avant l'étude, permettant une réévaluation dosimétrique. Ce recueil et cette mémorisation des informations constituent les premières étapes dans l'évaluation à long terme des thérapeutiques, maintenant indispensable, dans de nombreuses spécialités médicales

\section{RÉFÉRENCES}

1. Shore RE. Issues and epidemiological evidence regarding radiation-induced thyroid cancer. Rad Res 1992 ; 131 : 98-111.

2. Holm LE, Dahlquist I, Israelssson A, Lundell G. Malignant thyroid tumors after iodine-131 therapy. A retrospective study. $N$ Engl J Med 1980 ; 303 : 188-91.

3. Holm LE, Wiklund KE, Lundell GE, et al. Thyroid cancer after diagnosis doses of iodine-131: a retrospective study. J Natl Cancer Inst $1988 ; 80: 1132-8$.

4. Gallc P. Effets pathologiques des rayonnements ionisants. In : Leclère J, Orgiazzi J, Rousset J, Schienger JL, Wemeau JL, eds. La Thyroidde. Paris : Expansion scientifique française, 1992 : 555-61.
5. Donaldson SS, Chassagne D, Sancho Garnier H, Beyer HP. Hemangiomas of infancy : results of ${ }^{90} \mathrm{Y}$ interstitial therapy : a retrospective study. Int I Radiat Oncol Biol Phys $1979 ; 5: 1-11$.

6. Fragu P, Lemarchand-Venencie F, Benhamou S, et al. Long-term effects in skin and thyroid after radiotherapy for skin angiomas : a French retrospective cohort study. Eur J Cancer 1991; 27 : 1215-22.

7. Sancho $\mathrm{H}$, Beyer HP, Skin tumors. In : Bloom HJG, Lemerle J, Neidart NK, Voute PA, eds. Cancer in Children. Clinical Management. Berlin: Springer Verlag, 1975: 273-83.

8. François $\mathrm{P}$, Beurtheret C, Dutreix A, De Vathaire F. A mathematical child phantom for calculation of dose to organs at risk. Med Phys $1988 ; 15: 328-32$.

9. Dutreix A, Marinello G, Wambersie A. Dosimétrie en curiethérapie. Paris: Masson, 1982.

10. François $\mathrm{P}$, Beurtheret $\mathrm{C}$, Dutreix A. Calculation of the dosc delivered outside the radiation beams. Med Phys 1988; 15 : 879-83.

11. Ron E, Modan B, Preston D, Alfandry E, Stovall M, Boice J. Thyroid neoplasia following low-dose irradiation in childhood. Rad Res 1989 ; 120 : 516-31.

12. Wang Z, Boice JD, Wei I, et al. Thyroid nodularity and chromosome aberrations among women in areas of high background radiation in China. $J$ Natl Cancer Inst $1990 ; 82: 478-85$

13. De Vathaire $F$, Fragu $P$, François $P$, et al. Long-term effects on the thyroid of irradiation for skin angiomas in childhood. Rad Res 1993 ; 133 : 381-6.

14. De Vathaire F. Etudes de cohorte pour l'estimation des risques cancérigènes des radiations ionisantes. La Lettre du Cancérologue 1992 ; 1 : 34-40.

\section{ADRESSES}

P. Fragu : directeur de recherche à l'Inserm. Unité de médecine nucléaire et Inserm U. 66, Institut Gustave-Roussy, 94805 Villejuif Cedex, France. F. De Vathaire : chargé de recherche à l'Inserm. Inserm U. 351, Institut Gustavc-Roussy, 94805 Villejuif Cedcx, France. P. François : radiophysicien des centres de lutte contre le cancer (CLCC). Service de radiothérapie, centre Claudius-Regaud, 31052 Toulouse Cedex, France. M. F. Avril : médecin des CLCC. Scrvice de dermatologie, Institut Gustave-Roussy, 94805 Villcjuif Cedex, France.

\section{TIRÉS A PART}

\section{P. Fragu.}

